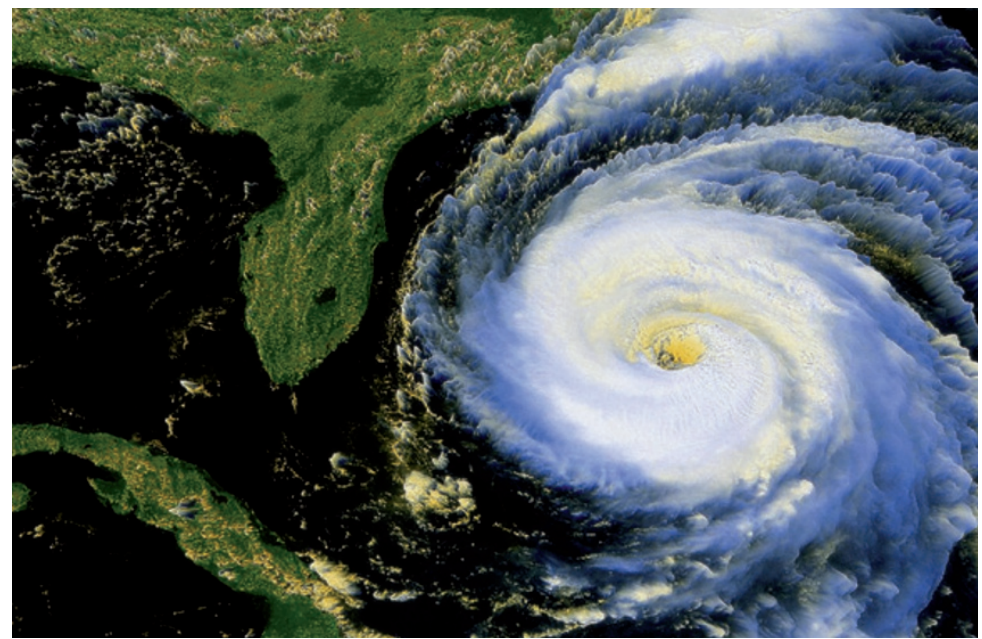

INFLAM MATION

\section{Trauma kicks up a storm}

alterations in the expression of systemic inflammatory response genes and compensatory antiinflammatory response genes occur rapidly and simultaneously following trauma
The current paradigm of the immune response to severe trauma is viewed as an early systemic inflammatory response followed by a temporary compensatory anti-inflammatory

recovery - this group comprised patients who developed severe multiple-organ dysfunction syndrome and recovered after day 14 , patients who had not recovered by day 28 and patients who died.

The authors found that the adaptive immunity. It is thought that serious complications are commonly associated with a 'second hit' (for example, infection or surgical stress) during this compensatory period that results in a more severe inflammatory response. Now, a study published in the Journal of Experimental Medicine proposes a new paradigm for the human immune response to severe trauma.

In this study, genome-wide expression analysis was performed on leukocytes that were isolated from the blood of 167 patients who had suffered severe blunt traumas. Leukocytes were first sampled within 12 hours of the trauma and then at several time points over the following 28 days. Of the 167 trauma patients, 41 had a complicated expression of $>80 \%$ of the leukocyte transcriptome was altered following severe blunt trauma, a phenomenon that the authors termed a 'genomic storm. The changes occurred rapidly (within 4-12 hours) and were maintained for days or weeks. Not surprisingly, the most upregulated genes were those involved in innate immunity, pathogen sensing and systemic inflammation, but also those with roles in compensatory anti-inflammatory responses (such as genes involved in interleukin-10 signalling). By contrast, most of the gene families that were downregulated to the greatest extent compared with control expression levels were involved in antigen presentation and $\mathrm{T}$ cell activation. The severity of injury and the volume of transfused blood minimally affected the gene expression patterns.

Of note, the genomic response pattern was remarkably similar in leukocytes from patients with severe burn injuries or who had received a low dose of a bacterial endotoxin, differing mainly in the duration of the response. This suggests the existence of a common response pattern in humans to severe injury and endotoxemia.

Next, the authors asked whether the gene expression profiles differ between patients with complicated and uncomplicated clinical recovery. Unexpectedly, they found that the gene expression profiles were not qualitatively different, but differed only in the magnitude and duration of the genomic storm. Indeed, very few genes or pathways were differentially regulated in patients with a complicated clinical recovery compared with those with an uncomplicated recovery.

Based on these data, the authors suggest a new model for the inflammatory paradigm in trauma. In this model, alterations in the expression of systemic inflammatory response genes and compensatory antiinflammatory response genes occur rapidly and simultaneously following trauma. Furthermore, it would appear that complicated recovery is not due to qualitative differences in the leukocyte transcriptome that would be consistent with a second hit. Rather, it is associated with greater and prolonged changes in gene expression, possibly owing to a lack of resolution of the inflammatory response.

Olive Leavy

ORIGINAL RESEARCH PAPER Xiao, W. et al.

A genomic storm in critically injured humans.J. Exp. Med. 21 Nov 2011 (doi:10.1084/jem.20111354) 